\title{
DECREMENTAL RESPONSE ON PROLONGED EXERCISE TEST IN A PATIENT WITH THYROTOXIC PERIODIC PARALYSIS
}

\author{
Piyush Ostwal $^{1}$, Maher Alshaheen ${ }^{2}$ \\ Correspondence: piyush.ostwal@gmail.com \\ ${ }^{1}$ Latrobe Regional Hospital, 10 Village Avenue, Traralgon, VIC, Australia \\ ${ }^{2}$ Bahrain Specialist Hospital, Building 2743, Road 2442, Juffair, Manama, Bahrain
}

\author{
Article History: \\ Received: $x x x, x x x x$ \\ Accepted: $x x x, x x x x$ \\ Published: January 1, 2022

\section{Cite this as:} \\ Ostwal P, Alshaheen M. \\ Decremental response on \\ prolonged exercise test in a \\ patient with thyrotoxic periodic \\ paralysis. Malang Neurology \\ Journal; 2022.8:64-67. DOI: \\ http://dx.doi.org/10.21776/ub.mnj \\ .2022 .008 .01 .13
}

\section{ABSTRACT}

Paralysis of acute onset often presents a diagnostic challenge for the assessing physician because of a large number of differential diagnosis and overlap of clinical features among them. Thyrotoxic periodic paralysis is an uncommon cause of acute weakness. In addition to serological tests, electromyography findings during prolonged exercise test are very helpful in confirming the diagnosis. Only a few case reports of thyrotoxic periodic paralysis have been published from Middle East and none of them have described this specific electrophysiological data. A man in his $20 \mathrm{~s}$ presented to us with acute onset weakness in both legs which was evaluated further and found to have hypokalemia. The work up for the etiology revealed thyrotoxic status and a final diagnosis of thyrotoxic periodic paralysis was established. The prolonged exercise test performed in this patient showed typical progressive decremental respsonse with nadir at 40 minutes after the exercise.

Keywords: Hypokalemic periodic paralysis, electromyography, thyrotoxicosis, nerve conduction

\section{Introduction}

Acute onset quadriparesis needs consideration of broad range of differential diagnoses. Careful history and examination is essential to work out the level of neurological insult which can be anywhere from brain down to the muscles. Hypokalemia is a known trigger for weakness attributable to muscles. Hypokalemia can be a consequence of either gastrointestinal/ renal potassium loss or intracellular shift of $\mathrm{K}^{+}$. A meticulous laboratory work up for the various etiologies of hypokalemia is warranted when faced with this clinical situation. Among these etiologies thyrotoxic periodic paralysis (TPP) has been recognized as a cause of recurrent paralytic attacks in Asian population. ${ }^{1}$ The presentation is not always obvious and a high index of suspicion is required to establish the diagnosis. ${ }^{2}$ Interestingly the presentation with TPP in more common in males despite hyperthyroidism being more prevalent in females. The clinical picture can vary in severity from mild weakness to severe paralysis. ${ }^{3}$ Apart from potassium and thyroid testing, electrophysiological testing with prolonged exercise test protocol is helpful in confirming the diagnosis during an acute attack of paralysis. In this test compound muscle action potential (CMAP) is measured serially after a period of sustained isometric exercise of the muscle. A progressive and slow decline in CMAP amplitude and area is characteristic feature of periodic paralysis. ${ }^{4}$ There are only sparse case reports of TPP from the Middle East region and none of them have described the detailed electrophysiological findings. ${ }^{5}$ Here we describe a patient who was diagnosed to have TPP and his electromyography showed characteristic decrement during prolonged exercise test.

\section{Case Report}

A 29-year-old Filipino gentleman presented with history of weakness and pain of both lower limbs of eight hours duration which started in the middle of night, when he wanted to go to toilet and found himself unable to stand and walk independently. He also had weakness and pain in left shoulder. He denied any numbness, tingling, bowel or bladder symptoms. There was no family history of similar illness. On examination there was mild diffuse smooth goiter, The higher mental function and cranial nerve examination was normal. On motor examination he had power grade $4 / 5$ in left shoulder, $3 / 5$ in both hips and 4/5 distally in the legs. There was no sensory loss. The deep tendon reflexes were normal. The planter was flexor. He was evaluated with MRI cervical spine which did not show any significant findings. His blood tests showed serum potassium of $3.1 \mathrm{mmol} / \mathrm{L}$ (normal $3.5-5.1 \mathrm{mmol} / \mathrm{L}$ ). The serum creatine kinase level was $303 \mathrm{U} / \mathrm{L}$ (normal 0 - 190 U/L). His electrocardiogram was normal. At this point, with a diagnosis of hypokalemic paralysis, he was treated with intravenous potassium and he showed marked recovery in the lower limb weakness over duration of six hours. On probing further he revealed that for past couple of months he used to have generalized bodyaches on the morning of first day at work following rest on weekend. 



A

B

Figure 1. Prolonged exercise test recording from abductor digiti minimi muscle stimulating the ulnar nerve at wrist. The recording starts from the top of panel A and continues into panel B from top to bottom. The voltage gain was $5 \mathrm{mV} /$ division and the sweep speed was $5 \mathrm{~ms} /$ division.

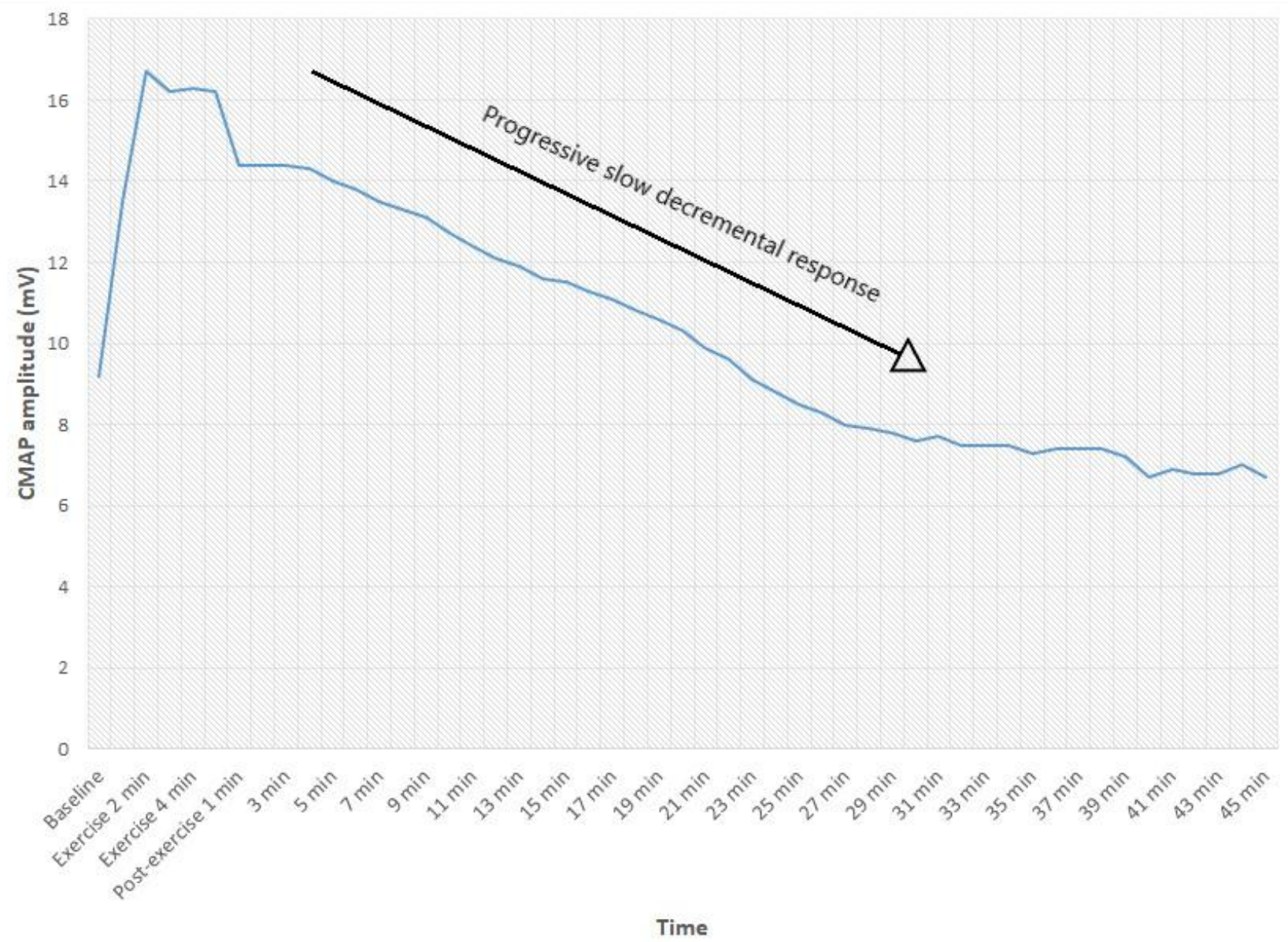

Figure 2. This line graph depicts compound muscle action potential (CMAP) amplitude (in mV) at various time points during prolonged exercise testing. CMAP was recorded from abductor digiti minimi muscle with stimulation of ulnar nerve at wrist. Note the initial rise in amplitude with exercise followed by gradual fall up to 40 minutes of resting. 
He did not have any exacerbation of symptoms after carbohydrate rich food, alcohol intake or on exposure to cold. On further evaluation the urine potassium level was $7.64 \mathrm{mmol} / \mathrm{L}$ excluding excessive urinary loss. The arterial blood gas results did not show any acid-base disturbance. The thyroid function tests revealed low TSH of 0.005 $\mu \mathrm{IU} / \mathrm{ml}$ (normal $0.270-4.200 \mu \mathrm{IU} / \mathrm{ml}$ ), high free T4 of $41.31 \mathrm{pmol} / \mathrm{L}$ (normal $12-22 \mathrm{pmol} / \mathrm{L}$ ) and high free T3 of17.82 pmol/L (normal $3.1-6.8 \mathrm{pmol} / \mathrm{L}$ ). His TSH receptor antibodies level was $2.97 \mathrm{IU} / \mathrm{L}$ (reference range 0 - $1.22 \mathrm{IU} / \mathrm{L})$, anti-thyroid peroxidase antibody level was $62.35 \mathrm{IU} / \mathrm{mL}$ (reference range $0-34 \mathrm{IU} / \mathrm{mL}$ ) and antithyroglobulin antibody level was $30.65 \mathrm{IU} / \mathrm{mL}$ (reference range $10-115 \mathrm{IU} / \mathrm{mL}$ ). His serum magnesium and phosphate levels were normal. The thyroid ultrasound showed normal size of the gland and echotexture. While he was recovering from weakness he was evaluated with electromyography. The routine nerve conduction study performed in all four limbs was normal. Needle electromyography did not show any evidence of myotonia. Further testing was done in accordance with the prolonged exercise test protocol. The recording was done from abductor digiti minimi muscle using the belly tendon configuration. Ulnar nerve was stimulated at the wrist using bipolar electrode stabilized with tape. Supramaximal stimulus was used throughout the test. For the test, the abductor digiti minimi was exercised as strongly as possible for five minutes with brief five seconds rests every 25 seconds to prevent ischemia. The compound muscle action potentials were recorded at baseline, every minute during the exercise and every minute after exercise till no further decrement was observed in Compound muscle action potential (CMAP) amplitude. The baseline - to - negative peak amplitude was measured and percent decrement was measured using the formula: [Peak - Nadir]/ Peak X 100. The lowest amplitude was recorded 40 minutes following exercise and the percent decrement was 59.9\%. (Figure 1 and figure 2)

Based on these clinical features and investigations, the final diagnosis was TPP associated with Grave's disease. He was started on propranolol and carbimazole for the definitive treatment of thyrotoxic state and prevention of paralytic attacks. On follow up at one month, he reported no further attacks of weakness. His creatine kinase, free T4 and free T3 levels had normalized.

\section{Discussion}

This case emphasizes the need to recognize hypokalemic paralysis in the setting of otherwise asymptomatic thyrotoxicosis. TPP is more common in Oriental Asians with reported incidence as high as $13 \%$ among thyrotoxic patients, whereas the incidence in other races in North America is estimated to be $0.1-0.2 \%$ of thyrotoxic patients. The average age of onset is around 30 years and is predominantly seen in males. Though seen most often in patients of thyrotoxicosis with Grave's disease, TPP has been reported with other hyperthyroid conditions like thyroiditis, toxic adenoma, toxic nodular goitre, TSHsecreting pituitary tumor, iodine-induced thyrotoxicosis (Jod-Basedow phenomenon), administration of radioactive iodine and amiodarone-induced thyrotoxicosis. The attacks of paralysis are precipitated by stress, alcohol intake or a carbohydrate-rich meal. ${ }^{6}$ There is only one previous case report of TPP from Bahrain and a few cases have been reported from surrounding Gulf countries. ${ }^{5,7}$

The hypokalemia in TPP results from shift of potassium to intracellular compartment due to in-appropriate activity of sodium potassium adenosine triphosphate $\left(\mathrm{N}^{+} / \mathrm{K}^{+}\right.$ATPase $)$ pump and Kir channels which impairs the membrane excitability and is reflected in reduced CMAPs on prolonged exercise testing. ${ }^{8}$ The CMAP amplitude rises briefly following exercise and progressively decreases up to 20 to 40 minutes post exercise. The drop in amplitude of greater than $40 \%$ is considered significant. ${ }^{4}$ In our case the lowest amplitude was recorded 40 minutes after stopping the exercise and was lower by $60 \%$ compared to the highest amplitude recorded after starting the exercise. The test is quite specific but does not distinguish between hypokalemic, hyperkalemic and thyrotoxic periodic paralyses. ${ }^{9}$ It helps in confirming the diagnosis in an objective way and in combination with short exercise test, can help in categorization of channelopathies. ${ }^{10}$

\section{Conclusion}

TPP should be considered in the differential diagnosis of acute onset weakness. The condition is especially prevalent in oriental Asian population. The electrophysiological evaluation with prolonged exercise test should be included as part of workup of such cases.

\section{Conflict of Interest}

There was no conflict of interest.

\section{Acknowledgement}

We thank Neuro-technologist Muniyappan for enthusiastic performance of the neurophysiological testing. We also thank Bahrain Specialist Hospital administration for the constant support and encouragement for research.

\section{References}

1. Chang C-C, Cheng C-J, Sung C-C, Chiueh T-S, Lee C-H, Chau T, et al. A 10-year analysis of thyrotoxic periodic paralysis in 135 patients: Focus on symptomatology and precipitants. Europe-an Journal of Endocrinology; 2013. 169:529-36.

DOI: $10.1530 / E J E-13-0381$

2. Bilha S, Mitu O, Teodoriu L, Haba C, Preda C. Thyrotoxic periodic paralysis: A misleading challenge in the emergency department. diagnostics (Basel) [Internet]; 2020 May 18 [cited 2020 Sep 29]. 10(5). Available from: https://www.ncbi.nlm.nih.gov/pmc/articles/PMC7277 936/

3. Iqbal QZ, Niazi M, Zia Z, Sattar SBA. A literature review on thyrotoxic periodic paralysis. Cureus; 2020. 12(8):e10108. DOI: 10.7759/cureus.10108

4. Simmons DB, Lanning J, Cleland JC, Puwanant A, Twydell PT, Griggs RC, et al. Long exercise test in periodic paralysis: A bayesian analysis. Muscle \& Nerve; 2019. 59(1):47-54. DOI: 10.1002/mus.2615 
5. Hakami O, Ahmad MM, Al Johani N. A case of nonfatal ventricular arrhythmia due to thyro-toxic periodic paralysis in a Saudi patient as an initial presentation of Graves' disease. Clin Med Insights Case Rep; 2016. 9:5-9. DOI: 10.4137/CCRep.S34560

6. Salih M, Duschek EJJ. Thyrotoxic periodic paralysis: An unusual presentation of hyperthyroidism. The Netherlands Journal of Medicine; 2017. 75(8):6. Avalaible from:

https://pubmed.ncbi.nlm.nih.gov/29219824/

7. Dilshad Siddiqui. Thyrotoxicosis presenting as hypokalemic periodic paralysis. Bahrain Medical Bulletin; 1998;20(2):58-60. Avalaible from: https://www.bahrainmedicalbulletin.com/june_1998/T hyrotoxicosis.pdf
8. Lin S-H, Huang C-L. Mechanism of thyrotoxic periodic paralysis. J Am Soc Nephrol; 2012. 23:9858. DOI: 10.1681/ASN.2012010046

9. Kuntzer T, Flocard F, Vial C, Kohler A, Magistris M, Labarre-Vila A, et al. Exercise test in mus-cle channelopathies and other muscle disorders. Muscle \& Nerve; 2000. 23:1089-94. DOI: 10.1002/10974598(200007)23:7<1089::aid-mus12>3.0.co;2-q

10. Fournier E, Arzel M, Sternberg D, Vicart S, Laforet P, Eymard B, et al. Electromyography guides toward subgroups of mutations in muscle channelopathies. Ann Neurol; 2004;56:650-61.

DOI: 10.1002/ana.20241 